the shear rate increases, the DNA molecules tend to align more towards the flow direction (see Fig. 4 legend).

Polymers extended in the direction of the shear flow have been qualitatively predicted by classical theories, and have been indirectly observed by using bulk birefringence ${ }^{3,4}$. Partial stretching of individual polymers in the direction parallel to the flow direction is due to the viscous drag exerted on the molecule ${ }^{5}$, which has been invoked to explain shear-thinning in polymer solutions ${ }^{1}$. However, the existence of vertical conformations (such as those shown in Fig. $2 \mathrm{a}-\mathrm{c}$ ) is unexpected, and is not considered by classical models of polymer physics ${ }^{5,6}$. Classically, it is assumed that, in the plane parallel to the velocity and the velocity-gradient directions, shear orients polymers at an angle $\leqslant 45^{\circ}$ with the flow direction. Shear may also induce tumbling of the polymers within such a plane. However, in the plane parallel to the velocity and the vorticity directions (the present plane of visualization; see Fig. 1), neither shear-induced alignment nor polymer tumbling can explain the existence of vertical conformations. Although fluid-mechanics models - such as Jeffery orbits describing the motion of axisymmetric rigid particles in a shear flow-may serve as limitingcase analyses $^{19-23}$, they cannot be directly applied to the dynamics of flexible polymers reported here. Furthermore, classical polymer theories predict ${ }^{5}$ that a flexible polymer becomes stretched and oriented when the Weissenberg number, $\mathrm{We}=\dot{\gamma} \tau$, is equal to or larger than unity. But here we find shear-induced deformation of flexible polymers can occur at Weissenberg numbers much smaller than unity (Figs 1-4).

Our experimental results show that conventional approaches, such as birefringence and light scattering, which measure only ensemble-averaged molecular parameters (that is, $\langle\theta\rangle,\langle l\rangle$ ), overlook the extremely rich dynamics of individual polymers under shear. Our observations also provide an insight into the conformational and orientational changes of polymers in a shear flow, and a basis for further theoretical modelling. The molecular-level approach to non-equilibrium polymer physics that we describe here could be readily extended to many other polymer systems, including entangled solutions of flexible polymers and semiflexible polymers such as $\operatorname{actin}^{24,25}$.

Received 21 October 1998; accepted 22 March 1999.

Munk, P. Introduction to Macromolecular Science (Wiley \& Sons, New York, 1989).

2. Hoffman, A., Ratner, B. \& Horbett, T. Polymers as Biomaterials (Plenum, New York, 1995).

Fuller, G. G. Optical Rheometry of Complex Fluids (Oxford Univ. Press, New York, 1995).

4. Janeschitz-Kriegl, H. Polymer Melt Rheology and Flow Birefringence (Springer, New York, 1983).

Doi, M. \& Edwards, S. F. The Theory of Polymer Dynamics (Clarendon, Oxford, 1986).

de Gennes, P. G. Scaling Concepts in Polymer Physics (Cornell Univ. Press, Ithaca, 1991).

de Gennes, P. G. Molecular individualism. Science 276, 199 (1997).

8. Matsumoto, M. et al. Direct observation of Brownian-motion of macromolecules by fluorescence microscope. J. Polym. Sci. 30, 779-783 (1992).

9. Perkins, T. T., Quake, S. R., Douglas, D. E. \& Chu, S. Relaxation of a single DNA molecule observed by optical microscopy. Science 264, 822-826 (1994).

10. Perkins, T. T., Smith, D. E. \& Chu, S. Single polymer dynamics in an elongational flow. Science $\mathbf{2 7 6}$ 2016-2021 (1997)

11. Schwartz, D. C. \& Koval, M. Conformational dynamics of individual DNA molecules during gel electrophoresis. Nature 338, 520-522 (1989).

12. Shivashankar, G. \& Libchaber, A. Single DNA molecule grafting and manipulation using a combined atomic force microscope and an optical tweezer. Appl. Phys. Lett. 7, 3727-3729 (1997).

13. Smith, D. E., Perkins, T. T. \& Chu, S. Dynamical scaling of DNA diffusion coefficients. Macromolecule 29, 1372-1373 (1996).

14. Smith, S. B., Finzi, L. \& Bustamante, C. Direct mechanical measurements of the elasticity of single DNA molecules by using magnetic beads. Science 258, 1122-1126 (1992)

15. Wirtz, D. Direct measurement of the transport properties of a single DNA molecule. Phys. Rev. Lett. 75, 2436-2439 (1995)

16. Morikawa, K. \& Yanagida, M. Visualization of individual DNA molecules in solution by ligh microscopy: DAPI staining method. J. Biochem. 89, 693-696 (1981)

17. Archer, L. A., Larson, R. G. \& Chen, Y. L. Direct measurement of slip in sheared polymer solutions. J. Fluid Mech. 301, 133-151 (1995).

18. Matsumoto, S., Morikawa, K. \& Yanagida, M. Light microscopic structure of DNA in solution studied by the $4^{\prime}, 6$-diamidinio-2-phenylindole staining method. J. Mol. Biol. 152, 501-516 (1981).

19. Jeffery, G. B. The motion of ellipsoidal particles immersed in a viscous fluid. Proc. R. Soc. Lond. A 102, 161-179 (1922)

20. Leal, L. G. \& Hinch, E. J. The effect of weak Brownian rotations on particles in shear flow. J. Fluid Mech. 46, 685-703 (1971)

21. Hinch, E. J. The deformation of a nearly straight thread in a shearing flow with weak Brownian motions. J. Fluid Mech. 75, 765-775 (1976).

22. Hinch, E. J. The distortion of a flexible inextensible thread in a shearing flow. J. Fluid Mech. 74, 317 333 (1976).

23. Hinch, E. J. Mechanical models of dilute polymer solutions in strong flows. Phys. Fluids 20, S22-S30 (1977)
24. Xu, J., Palmer, A. \& Wirtz, D. Rheology and microrheology of semiflexible polymer solutions: actin filament networks. Macromolecules 31, 6486-6492 (1998).

25. Ostap, E. M., Yanagida, T. \& Thomas, D. D. Orientational distribution of spin-labeled actin oriented by flow. Biophys. J. 63, 966-975 (1992).

Acknowledgements. We thank M. Ferro, L. Archer, J. Harden, D. Lavan, and J. van Zanten for discussions. This work was supported by NASA (D.W.), ACS-PRF (D.W.), NSF (D.W.), the Whitaker Foundation (D.W.), Merck, Inc. (D.W.) ARO (G.B.), and IMRE (G.B.)

Correspondence and requests for materials should be addressed to D.W. (e-mail: wirtz@jhu.edu).

\section{Tuning bilayer twist using chiral counterions}

\author{
R. Oda ${ }^{\star} \dagger$, I. Huc ${ }^{\star}$, M. Schmutz $\ddagger$, S. J. Candau $\dagger$ \\ \& F. C. MacKintosh $₫ \dagger$
}

* Institut Européen de Chimie et Biologie, ENSCPB Av. Pey Berland, BP 108, 33402 Talence Cedex, France

$\dagger$ Laboratoire de Dynamique des Fluides Complexes, 3 Rue de l'Université, 67000 Strasbourg, France

¥Institut de Génétique et de Biologie Moléculaire et Cellulaire, INSERM/CNRS/ULP, BP 163, 67404 Illkirch Cedex, France

$\$$ Department of Physics, University of Michigan, Ann Arbor,

Michigan 48109-1120, USA

From seashells to DNA, chirality is expressed at every level of biological structures. In self-assembled structures it may emerge cooperatively from chirality at the molecular scale. Amphiphilic molecules, for example, can form a variety of aggregates and mesophases that express the chirality of their constituent molecules at a supramolecular scale of micrometres (refs 1-3). Quantitative prediction of the large-scale chirality based on that at the molecular scale remains a largely unsolved problem. Furthermore, experimental control over the expression of chirality at the supramolecular level is difficult to achieve ${ }^{4-7}$ : mixing of different enantiomers usually results in phase separation $^{18}$. Here we present an experimental and theoretical description of a system in which chirality can be varied continuously and controllably ('tuned') in micrometre-scale structures. We observe the formation of twisted ribbons consisting of bilayers of gemini surfactants (two surfactant molecules covalently linked at their charged head groups). We find that the degree of twist and the pitch of the ribbons can be tuned by the introduction of opposite-handed chiral counterions in various proportions. This degree of control might be of practical value; for example, in the use of the helical structures as templates for helical crystallization of macromolecules ${ }^{8,9}$.

Gemini surfactants, consisting of two identical (twin) surfactants joined by a hydrocarbon spacer of variable length, have been shown to have properties that are unusual compared to those of simple surfactants and lipids ${ }^{10}$. Cationic gemini surfactants having chiral counterions such as L-tartrate (Fig. 1) form gels in both water and some organic solvents ${ }^{11}$ by creating extended networks of the multilamellar twisted ribbons reported here (Fig. 2, helix B). A feature of this system is that the chirality comes from the counterion rather than from the amphiphile itself, which allows us to both adjust the pitch and to introduce excess chirality in the form of sodium tartrate salts.

Similar structures to those that we observe are found for diacetylenic lipids, bile and glutamates ${ }^{1,2}$, which form long helical strips of membranes with exposed edges (Fig. 2, helix A). However, these helical ribbons are unstable: they evolve into tubules (Fig. 2) consisting of a bilayer (or multilayer) membrane of amphilic molecules wrapped in a cylinder which exhibits a 'barber's-pole' pattern on its surface as evidence of its chiral origin.

The twisted ribbons that we observe have several original features. Geometrically, their saddle-like curvature differs from the cylind- 


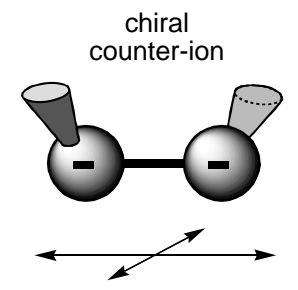

anisotropic

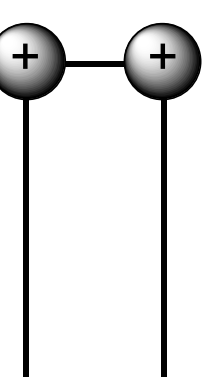
head group

$$
\overbrace{\mathrm{COO}_{2} \mathrm{C}}^{\mathrm{OH}}
$$

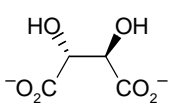

D-tartrate

L-tartrate<smiles>O=C([O-])[C@@H](O)[C@@H](O)[C@@H](O)[C@@H](O)C(=O)[O-]</smiles><smiles>[O-][O+]=[Os]</smiles>

D-glucarate

L-malate

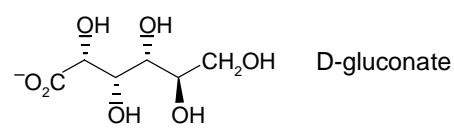

$\begin{array}{ll}\mathrm{C}_{\mathrm{n}=12-22} & 2 \mathrm{X}^{-}=\mathrm{D} \text {-tartrate } \\ & 2 \mathrm{X}^{-}=\text {L-tartrate } \\ & 2 \mathrm{X}^{-}=\mathrm{D} \text {-glucarate } \\ & 2 \mathrm{X}^{-}=2 \text { D-glace }\end{array}$

Figure 1 Cationic gemini amphiphiles having chiral counterions. Shown are the structures of ethylene-1,2-bis(dimethylalkylammonium) surfactants with various chiral anions. The nomenclature $n-2-n$ refers to the number of carbon atoms of the first hydrocarbon chain, the ethylene spacer, and the second hydrocarbon chain, respectively. Only the tartrate derivatives form twisted ribbons, the pitch and width of which decreases for shorter hydrocarbon chains. When the counterion is L-malate, which lacks one of the hydroxy groups of tartrate, hydrogen-bonding between the counterions is weakened and only flat bilayers are observed despite the chirality of the components. When using gluconate or glucarate as counterions, extended hydrogen bonding remains possible, but the affinity of the anions for the surfactant head groups is decreased due to the larger distance between the negative charges. Again, these ions do not cause a twist in the amphiphilic bilayers.

rical curvature of helical ribbons. Also, in contrast with the tubuleforming systems, we observe no evolution towards closed chiral aggregates. The twisted ribbons may be thermodynamically stable, as is predicted for highly chiral systems by the model described below. Most importantly, we demonstrate that the chirality of the microstructures can be continuously varied by mixing of counterion enantiomers.

We find that the pure 16-2-16 L-tartrate consistently forms twisted ribbons of the same handedness, whilst the D-enantiomer forms ribbons of opposite handedness (the 16-2-16 nomenclature is defined in Fig. 1 legend). However, these enantiomers do not undergo a lateral phase separation into helices with opposite chiralities when mixed in arbitrary proportions. Instead, they mix homogeneously and form helices with a continuous variation of twist period and width (Fig. 3). An increase of the enantiomeric excess (e.e.) causes a decrease of the twist pitch from infinite (flat ribbons) to $200 \mathrm{~nm}$ (Table 1): e.e. is defined as the relative concentration difference $\left(\phi_{\mathrm{L}}-\phi_{\mathrm{D}}\right) /\left(\phi_{\mathrm{L}}+\phi_{\mathrm{D}}\right)$, from 0 (racemic) to 1 (pure L-tartrate). On increasing the enantiomeric excess, we also see a decrease of the mean width of the ribbons from $\sim 400 \mathrm{~nm}$ to $40 \mathrm{~nm}$, and their period and width seem to become more regular (Table 1). On addition of the salt sodium L-tartrate, a helix of 16-216 L-tartrate twists even further (Fig. 3d). The decrease of the pitch and width eventually reaches a limit $(100 \mathrm{~nm}$ and $20 \mathrm{~nm}$, respectively) at $\sim 3$ equiv. of salt added, presumably as the cationic bilayers become saturated with chiral anions (Table 1). Thus, tuning the geometrical parameters of these helices can be performed using very simple means.

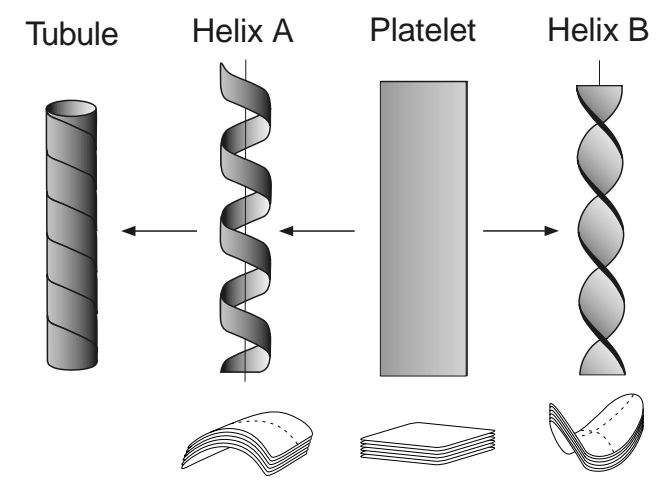

Figure 2 Schematic representation of helical and twisted ribbons. Top, platelet or flat ribbon. Helical ribbons (helix A), precursors of tubules, feature inner and outer faces. Twisted ribbons (helix B), formed by some $n-2-n$ tartrate surfactants, have equally curved faces and a C2 symmetry axis. Bottom, the consequences of cylindrical and saddle-like curvatures in multilayered structures. In a stack of cylindrical sheets, the contact area from one layer to the next varies. This is not the case for saddle-like curvature, which is thus favoured when the layers are coordinated.

How does the shape of the twisted ribbons arise from the particular molecular structure of the amphiphiles? To answer this, we examined the properties of structural variants of 16-2-16 tartrate, and came to the following conclusions.

First, the absence of twist for the malate derivative (Fig. 1), and the properties of the gels in organic solvents ${ }^{11}$, strongly support extensive hydrogen-bonding of tartrate counterions between consecutive bilayers. For simple geometric reasons, such interlayer coordination tends to favour a saddle-like curvature over that of a cylinder (see Fig. 2 legend).

Second, in water the twisted ribbons are stable for gemini surfactants with hydrocarbon chains ranging from 14 to 16 carbons. For shorter hydrocarbon chains, only micelles form for concentrations at least up to $40 \%$. At 18 carbons, helical ribbons form instead of twisted ribbons, presumably as a result of the elevated chain melting temperature and the consequent solid phase, which will favour a cylindrical shape.

Third, the ability of the amphiphile to form such anisotropic aggregates (having ribbons with both long and short sides) appears to be directly linked to its dimeric character. For instance, the monomeric counterpart, cetyl trimethyl ammonium tartrate, fails to form any ribbon-like structure, whilst gemini surfactants having simple bromides as counterions form flat ribbons similar to those of Fig. 3a (ref. 12). Furthermore, these highly anisotropic structures

\begin{tabular}{|c|c|c|c|}
\hline e.e. & $\begin{array}{c}T \\
(\mu \mathrm{m})\end{array}$ & $\begin{array}{c}W \\
(\mu \mathrm{m})\end{array}$ & $T / W$ \\
\hline 0 & $\infty$ & $0.40 \pm 0.28$ & \\
\hline 0.2 & $5.0 \pm 1.5$ & $0.12 \pm 0.046$ & $43 \pm 5.2$ \\
\hline 0.33 & $2.6 \pm 0.9$ & $0.11 \pm 0.047$ & $26 \pm 7.0$ \\
\hline 0.5 & $1.0 \pm 0.5$ & $0.06 \pm 0.016$ & $17 \pm 4.7$ \\
\hline 1 & $0.2 \pm 0.024$ & $0.04 \pm 0.006$ & $4.8 \pm 0.51$ \\
\hline $1^{*}$ & $0.12 \pm 0.007$ & $0.02 \pm 0.002$ & $7.0 \pm 0.90$ \\
\hline $1 \dagger$ & $0.115 \pm 0.01$ & $0.02 \pm 0.002$ & $5.6 \pm 0.86$ \\
\hline $1 \neq$ & $0.115 \pm 0.006$ & $0.02 \pm 0.002$ & $5.9 \pm 0.63$ \\
\hline
\end{tabular}
strongly suggest a long-range alignment of the constituent

Shown are values of enantiomeric excess (e.e., defined as $\left.\left(\phi_{L}-\phi_{D}\right) /\left(\phi_{L}+\phi_{D}\right)\right)$ and the mean values and standard deviations of ribbon period $(T)$, width $(W)$, and period/width $(T / W)$, for different proportions of $L$ and $D$ enantiomers of 16-2-16 tartrate. Statistics were derived from 10-20 measurements for each sample.

* In the presence of 1 equiv. of sodium L-tartrate.

$t$ In the presence of 3 equiv. of sodium L-tartrate.

$\ddagger$ In the presence of 10 equiv. of sodium L-tartrate. 
molecules, which may be correlated with a hydrogen-bonded network of tartrate ions ${ }^{11}$.

In the model we propose, orientational order of the molecules within the membrane together with the chirality of the constituents provide a natural explanation for the observed twisted-ribbon shape, and can also account for the observed trends in the pitch and width of the ribbons on variation of the enantiomeric excess. In a membrane composed of gemini surfactants, an anisotropic fluidlike arrangement of head groups would be analogous to nematic order in bulk liquid crystals ${ }^{13}$.

The shape of the membrane is described by a curvature tensor $K_{i j}$, whose eigen values $c_{1}$ and $c_{2}$ are the principal curvatures along two orthogonal directions in the membrane ${ }^{14}$. Including both bending energies and the coupling of membrane shape to the assumed nematic field ${ }^{13} Q_{i j}=S\left(n_{i} n_{j}-\frac{1}{2} \delta_{i j}\right)$, the free energy per unit area of membrane is given by ${ }^{15,16}$ :

$$
\begin{aligned}
f= & \frac{1}{2} \kappa\left(\operatorname{Tr} K_{i j}\right)^{2}+\bar{\kappa} \operatorname{Det} K_{i j}+\lambda \epsilon_{i j} Q_{j k} K_{k i}+\frac{1}{2} \kappa^{\prime} Q_{i j} K_{j k} K_{k i} \\
= & \frac{1}{2} \kappa\left(c_{1}+c_{2}\right)^{2}+\bar{\kappa}\left(c_{1} c_{2}\right)+\lambda S \sin (2 \theta)\left(c_{1}-c_{2}\right) \\
& +\frac{1}{2} \kappa^{\prime} S \cos (2 \theta)\left(c_{1}+c_{2}\right)\left(c_{1}-c_{2}\right)
\end{aligned}
$$
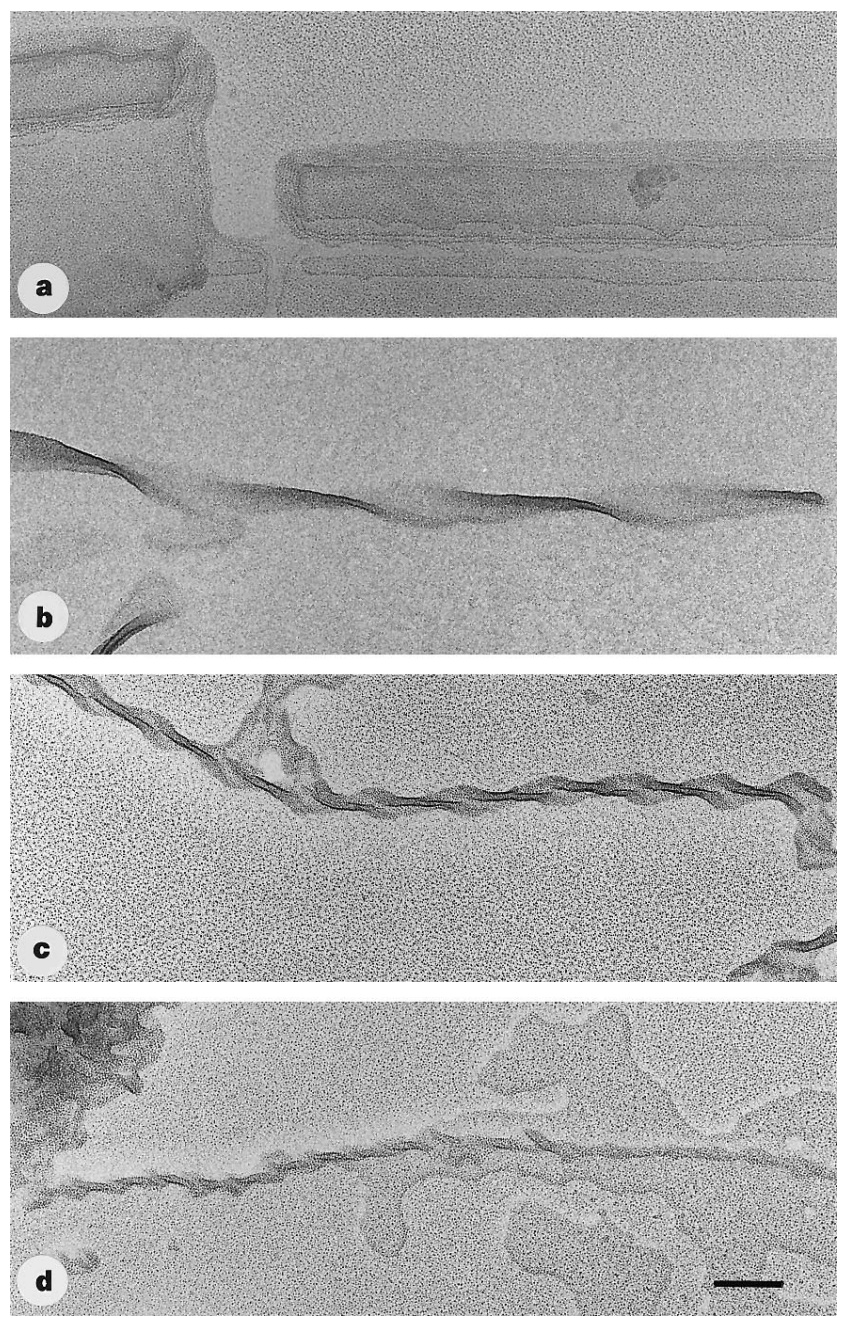

Figure 3 TEM images of the ribbons. Shown are representative twisted ribbons formed by 16-2-16 tartrate at $0.1 \%$ in water for various values of $\left(\phi_{L}-\phi_{D}\right) /\left(\phi_{L}+\phi_{D}\right)$. a, 0 (racemate); $\mathbf{b}, 0.5 ; \mathbf{c}, 1$ (pure L); $\mathbf{d}, 1$ (pure L) in the presence of 1 equiv. of sodium L-tartrate. Scale bar, $100 \mathrm{~nm}$.
Here, the coupling $\lambda$ characterizes the degree of chirality of the system (which should be proportional to the enantiomeric excess), $\theta$ is the angle that the molecular orientation makes with respect to the principle curvature axes, $\epsilon_{i j}$ is the antisymmetric tensor and $\kappa$, $\bar{\kappa}$ and $\kappa^{\prime}$ represent the mean, gaussian and anisotropic bending stiffness. Finally, we take into account the energy cost of the exposed edges of the ribbons by introducing an excess free energy $\gamma$ per unit length of edge. This model assumes that the degree of nematic order $S$ is fixed, by, for example, detailed molecular packing. Otherwise, it represents the most general continuum model that includes the curvature free-energy terms through quadratic order. It is thus expected to be valid provided that the membrane curvature is not too large (for example, on the molecular scale).

The analysis and results of this model will be described in greater detail elsewhere. Here we summarize the most relevant results for our experiments. First, for a given width of ribbon, the optimal shape (obtained by minimizing the bulk free energy $f$ with respect to shape) for chiral systems is that of the twisted ribbon with saddlelike curvature (specifically, $c_{1}=-c_{2}$ ) and the nematic director oriented at $45^{\circ}$ with respect to the curvature axes. For a fluid membrane, cylindrical curvature-either as a complete tubule, or as a helical ribbon precursor to a tubule-is not expected. In contrast, solid-like order would strongly oppose saddle-like curvature and favour cylindrical curvature, as is observed in tubuleforming systems ${ }^{17}$.

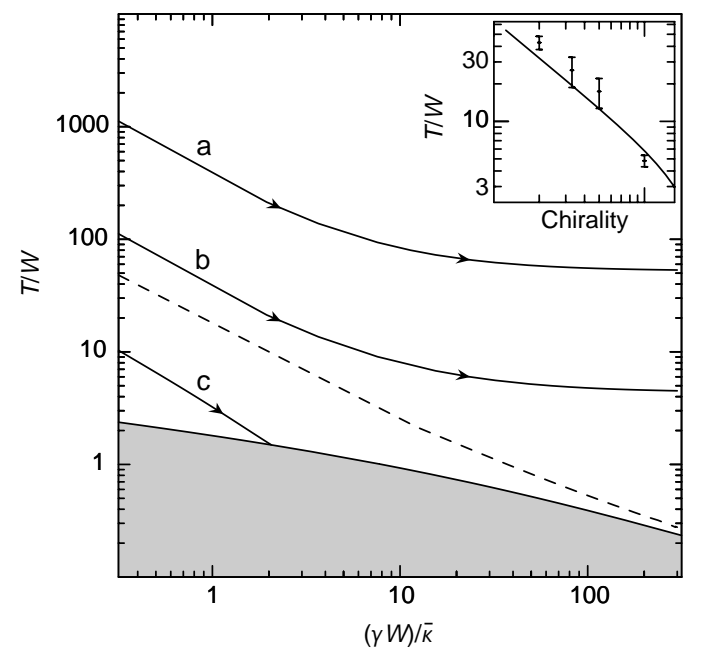

Figure 4 Predicted aspect ratio of twisted ribbons. Main figure, the calculated ratio of period to width, $T / W$, as a function of reduced width $\gamma W /|\bar{\kappa}|$. This is shown for increasing values of $\mu=\lambda S / \gamma$, which is proportional to the enantiomeric excess. Curve a is for $\mu=0.02$; $\mathrm{b}$ is for $\mu=0.2$; and $\mathrm{c}$ is for $\mu=2$. For a given $\mu$, only solutions lying along the corresponding line are possible. However, these are typically non-equilibrium solutions, evolving as shown by the arrows towards wider structures, which reduce the total edge energy. For narrow ribbons, the twist period $T \propto 1 / \mu$ is constant. For $\mu<1 / 2$ (dashed line) no equilibrium solutions are possible, although $T$ and $W$ become strongly correlated: the ratio $T / W$ approaches a value near $1 / \mu$, even though polydisperse distributions of both $W$ and $T$ are expected. For $\mu>1 / 2$, the ribbons grow only until fixed, equilibrium values of $T$ and $W$ (shown by the filled circle) are reached. The equilibrium structures correspond to the upper boundary of the shaded region, within which no stable solutions are predicted. For all but a very narrow range of $\mu$ near the critical value of $1 / 2$, these are characterized by $T / W$ of the order of 1 . Inset, Measured ratio $T / W$ (points with error bars representing standard deviations of 10-20 samples for ribbons of mixtures of 16-2-16 $\mathrm{D}$ and $\mathrm{L}$ tartrate, without any salt added. Enantiomeric excess (chirality) ranges from 0.2 to 1.0 (pure enantiomer). The curve represents the predicted steady-state values of $T / W$ as a function of chirality $\mu=\lambda S / \gamma$. The precise relationship of the enantiomeric excess to $\mu$ involves an unknown constant of proportionality, which has been chosen to fit the data. 
As depicted in Fig. 4, minimization of the total free energy per surfactant predicts equilibrium ribbons if the chirality or enantiomeric excess is large enough (specifically, if $\lambda S \geqslant \gamma / 2$ ). Thus, despite the inevitable excess free energy associated with the edges, these ribbons may not grow beyond a certain preferred width $W$. In this case, the twist period $T$ and ribbon width $W$ are expected to vary inversely with the enantiomeric excess, to be well-defined (for example, their distributions are expected to be monodisperse), and the ratio $T / \mathrm{W}$ is expected to be of the order of 1 , and nearly independent of the enantiomeric excess. Before these equilibrium structures are attained, however, it is expected that $T$ varies inversely with the enantiomeric excess and is uncorrelated with $W$. Thus, the aspect ratio and monodispersity that we observe for ribbons formed by the pure 16-2-16 L-tartrate suggest that these are close to equilibrium.

The non-equilibrium structures predicted for weakly chiral systems (specifically, if $\lambda S \geqslant \gamma / 2$ ) are expected to exhibit correlated values of $T$ and $W: T / W$ varies inversely with enantiomeric excess, although $T$ and $W$ are expected to have polydisperse distributions. This corresponds well to the twisted ribbons formed by mixtures of $\mathrm{D}$ and $\mathrm{L}$ enantiomers, which exhibit such correlated, though polydisperse, geometrical parameters, and large $T / W$ ratios. The observed stability for these helices probably results from kinetic effects which limit their growth.

Thus, on the basis of molecular parameters such as chirality and anisotropy of the surfactant polar heads, this simple yet general model predicts that the observed twisted-ribbon shape is optimal, and that both the pitch and the width are expected to follow the trends that we observe on varying the chirality. Also important are the apparently qualitatively different behaviours for strongly and weakly chiral systems. The experimental observations and the theoretical model reported here may open the prospect of creating stable structures of variable pitch in amphiphilic bilayer systems.

Received 4 January; accepted 24 March 1999

1. Schnur, J. M. Lipid tubules: a paradigm for molecularly engineered structures. Science 262, 1669-1676 (1993).

2. Fuhrhop, J.-H. \& Helfrich, W. Fluid and solid fibers made of lipid molecular bilayers. Chem. Rev. 93, $1565-1582(1993)$

3. Rowan, A. E. \& Nolte, R. J. M. Helical molecular programming. Angew. Chem. Int. Edn 37, 63-68 (1998).

4. Chung, D. S., Benedek, G. B., Konikoff, F. M. \& Donovan, J. M. Elastic free energy of anisotropic helical ribbons as metastable intermediates in the crystallization of cholesterol. Proc. Natl Acad. Sci. USA 90, 11341-11345 (1993).

. Spector, M. S. et al. Controlling the morphology of chiral lipid tubules. Langmuir 14, 3493-3500 (1998).

6. Prins, L. J., Huskens, J., de Jong, F., Timmerman, P. \& Reinhoudt, D. N. Complete asymmetric induction of supramolecular chirality in a hydrogen-bonded assembly. Nature 398, 498-502 (1999). . Yashima, E., Maeda, K. \& Okamoto, Y. Memory of macromolecular helicity assisted by interaction with achiral small molecules. Nature 399, 449-451 (1999).

8. Ringler, P., Muller, W., Ringsdorf, H. \& Brisson, A. Functionalized lipid tubules as tools for helical crystallization of proteins. Chem. Eur. J. 3, 620-625 (1997).

9. Wilson-Kubalek, E. M., Brown, R. E., Celia, H. \& Milligan, R. A. Lipid nanotubes as substrates for helical crystallization of macromolecules. Proc. Natl Acad. Sci. USA 95, 8040-8045 (1998).

10. Zana, R. \& Talmon, Y. Dependence of aggregate morphology on structure of dimeric surfactants. Nature 362, 228-230 (1993).

11. Oda, R., Huc, I. \& Candau, S. J. Gemini surfactants as new, low molecular weight gelators of organic solvents and water. Angew. Chem. Int. Edn 37, 2689-2691 (1998).

12. Oda, R. et al. Elongated aggregates formed by cationic gemini surfactants. Langmuir 15, 2384-2390 (1999).

13. de Gennes, P. G. \& Prost, J. The Physics of Liquid Crystals 2nd edn (Clarendon, Oxford, 1993).

14. Helfrich, W. Elastic properties of lipid bilayers: theory and possible experiments. Z. Naturforsch. C 28, 693-703 (1973)

15. Helfrich, W. \& Prost, J. Intrinsic bending force in anisotropic membranes made of chiral molecules. Phys. Rev. A 38, 3065-3068 (1988).

16. Selinger, J. V., MacKintosh, F. C. \& Schnur, J. M. Theory of cylindrical tubules and helical ribbons of chiral lipid membranes. Phys. Rev. E 53, 3804-3818 (1996).

17. Thomas, B. N., Safinya, C. R., Plano, R. J. \& Clark, N. A. Lipid tubule self-assembly: length dependence on cooling rate through a first-order phase transition. Science 267, 1635-1638 (1995).

18. Singh, A. et al. Lateral phase separation based on chirality in a polymerizable lipid and its influence on formation of tubular microstructures. Chem. Phys. Lipids 47, 135-148 (1988).

Acknowledgements. This work was supported by the CNRS, INSERM, l'Hôpital Universitaire de Strasbourg, and Rhône-Poulenc. F.C.M. was supported in part by the CNRS, the Deutsche Forschungsgemeinschaft, the National Science Foundation, and the Petroleum Research Fund (administered by the ACS).

Correspondence and requests for materials should be addressed to R.O. or I.H. (e-mail: reiko.oda@iecbpolytechnique.u-bordeaux.fr)

\section{Causes of twentieth-century temperature change near the Earth's surface}

\author{
Simon F. B. Tett*, Peter A. Stott ${ }^{\star}$, Myles R. Allen $\dagger$, \\ William J. Ingram ${ }^{\star} \&$ John F. B. Mitchell ${ }^{\star}$
}

\author{
* Hadley Centre for Climate Prediction and Research, Meteorological Office, \\ London Road, Bracknell, Berkshire RG12 2SY, UK \\ $\dagger$ Space Science Department, Rutherford Appleton Laboratory, \\ Chilton, OX11 OQX, UK and Department of Physics, Univeristy of Oxford
}

Observations of the Earth's near-surface temperature show a global-mean temperature increase of approximately $0.6 \mathrm{~K}$ since 1900 (ref. 1), occurring from 1910 to 1940 and from 1970 to the present. The temperature change over the past 30-50 years is unlikely to be entirely due to internal climate variability ${ }^{2-4}$ and has been attributed to changes in the concentrations of greenhouse gases and sulphate aerosols ${ }^{5}$ due to human activity. Attribution of the warming early in the century has proved more elusive. Here we present a quantification of the possible contributions throughout the century from the four components most likely to be responsible for the large-scale temperature changes, of which two vary naturally (solar irradiance and stratospheric volcanic aerosols) and two have changed decisively due to anthropogenic influence (greenhouse gases and sulphate aerosols). The patterns of time/space changes in near-surface temperature due to the separate forcing components are simulated with a coupled atmosphere-ocean general circulation model, and a linear combination of these is fitted to observations. Thus our analysis is insensitive to errors in the simulated amplitude of these responses. We find that solar forcing may have contributed to the temperature changes early in the century, but anthropogenic causes combined with natural variability would also present a possible explanation. For the warming from 1946 to 1996 regardless of any possible amplification of solar or volcanic influence, we exclude purely natural forcing, and attribute it largely to the anthropogenic components.

The coupled model we use is HadCM2 (refs 6, 7), which has a horizontal resolution of $2.5^{\circ}$ in latitude by $3.75^{\circ}$ in longitude, 19 atmospheric and 20 oceanic levels, and a flux correction. Its climate sensitivity to a doubling of the atmospheric concentration of $\mathrm{CO}_{2}$ is estimated to be $3.3 \mathrm{~K}$ (C. A. Senior, personal communication).

The main radiative forcings of climate since 1850 are likely to be anthropogenic changes in well-mixed greenhouse gases and tropospheric aerosols (mainly sulphate), and natural changes in solar irradiance and in stratospheric aerosol due to volcanic activity ${ }^{8}$. We compare observations ${ }^{1}$ of 10 -year mean near-surface temperature changes over five 50-year periods (1906-56, 1916-66, ..., 1946-96) with simulations of HadCM2 forced by the following factors (see also section 1 of Supplementary Information):

'G'. Changes in well-mixed greenhouse gases from 1860 to $1996^{8,9,10}$ expressed as equivalent $\mathrm{CO}_{2}$.

'GS'. As G but also with changes in surface albedo ${ }^{11}$ representing the effects of anthropogenic sulphate aerosols from 1860 to $1996^{6,9,10}$ derived from an atmospheric chemistry model ${ }^{12}$. We assume that this albedo represents both the direct and indirect ${ }^{11,13,14}$ effects of sulphate aerosols. When we consider both G and GS we define a further signal S, the pure sulphate signal, as GS - G (see section 9 of Supplementary Information).

'Vol'. Changes in stratospheric volcanic aerosols ${ }^{15}$ from 1850 to 1996. 'Sol'. Changes in total solar irradiance from 1700 to 1996 based on proxy data ${ }^{16}$ for $1700-1991$ and extended to 1996 using satellite observations ${ }^{17}$. 